\title{
HÁBITOS ALIMENTARIOS Y ACTIVIDAD FÍSICA EN ADULTOS MAYORES CON ENFERMEDAD CRÓNICA
}

\author{
EATING HABITS AND PHYSICAL ACTIVITY IN OLDER ADULTS \\ WITH CHRONIC DISEASE
}

\section{HÁBITOS ALIMENTARES E ATIVIDADE FÍSICA EM IDOSOS COM DOENÇAS CRÔNICAS}

\author{
Martha Elba Salazar-Barajas* \\ Bertha Cecilia Salazar-González** \\ Hermelinda Ávila-Alpirez ${ }^{* * *}$ \\ Jesús Alejandro Guerra Ordóñez**** \\ Juana María Ruiz Cerino***** \\ Tirso DurÁn-BADILlo******
}

\begin{abstract}
RESUMEN
Objetivo: Determinar los factores relacionados con hábitos alimentarios y actividad física en adultos mayores con enfermedad crónica. Material y Método. Estudio correlacional de corte transversal en 200 adultos mayores de Matamoros, Tamaulipas, México. El trabajo de campo se realizó en el primer semestre de 2019 y mediante un cuestionario ex profeso, se midieron los hábitos alimentarios y actividad física. Se usaron medidas de tendencia central y dispersión para variables continuas; frecuencias y porcentajes para variables cualitativas; prueba de Kolmogorov-Smirnov con corrección de Lilliefors para determinar distribución de variables numéricas; coeficientes de correlación de Spearman para relaciones entre las variables sociodemográficas, enfermedad crónica, hábitos alimenticios y actividad física. El estudio fue aprobado por los comités de ética e investigación (FAEN-D-912). Resultados: El consumo de refrescos $(p=, 039)$, legumbres $(p=, 001)$ y pescado $(p=, 005)$ se relacionó con la escolaridad; la ingesta de agua $(p=, 007)$, legumbres $(p<, 001)$ y escolaridad $(p<, 001)$
\end{abstract}

*Doctora en Ciencias de Enfermería. Unidad Académica Multidisciplinaria, Matamoros. Universidad Autónoma de Tamaulipas, México. ORCID: https://orcid.org/0000-0001-6230-2547 Email: msalazar@docentes.uat.edu.mx

**PhD, Facultad de Enfermería. Universidad Autónoma de Nuevo León, México. ORCID: https://orcid.org/0000-0002-66108052 Email: bceci195@hotmail.com

***Doctora en Enfermería y Cultura de los Cuidados. Unidad Académica Multidisciplinaria, Matamoros. Universidad Autónoma de Tamaulipas, México. ORCID: http://orcid.org/0000-0001-5286-5944 Email: havila@docentes.uat.edu.mx

**** Doctor en Ciencias de Enfermería. Unidad Académica Multidisciplinaria, Matamoros. Universidad Autónoma de Tamaulipas, México. ORCID: https://orcid.org/0000-0002-4587-3214 Email: jesus.guerra@docentes.uat.edu.mx

***** Doctora en Metodología de la Enseñanza. Unidad Académica Multidisciplinaria, Matamoros. Universidad Autónoma de Tamaulipas, México. ORCID: https://orcid.org/0000-0002-0541-2464 Email: jruizc@docentes.uat.edu.mx

******* Doctor en Metodología de la Enseñanza. Unidad Académica Multidisciplinaria, Matamoros. Universidad Autónoma de Tamaulipas, México. ORCID: https://orcid.org/0000-0002-7274-3511 Email: tirdb27@hotmail.com Autor de correspondencia 
se relacionó con la edad; la caminata por semana $(p<, 001)$ y minutos que dedican a caminar $(p<, 001)$ se relacionó con el consumo de agua. Conclusiones. La alimentación y la actividad física en los adultos mayores son importantes para la prevención y control de las enfermedades crónicas y otro tipo de enfermedades. Los resultados de este estudio reflejan la urgencia de intervenir en etapas tempranas para enfatizar hábitos de una alimentación balanceada y la práctica regular de actividad física asociada a la salud.

Palabras clave: Conducta alimentaria; Actividad física asociada a la salud; Adulto mayor.

\begin{abstract}
Objective: To determine the factors related to eating habits and physical activity in older adults with chronic disease. Material and Method: Cross-sectional correlational study, carried out with 200 older adults from Matamoros, Tamaulipas State, Mexico. The field work was conducted in the first semester of 2019 and eating habits as well as physical activity were measured using an ex profeso questionnaire. Different measures were used: central tendency and dispersion for continuous variables; frequencies and percentages for qualitative variables; Kolmogorov-Smirnov test with Lilliefors correction to determine distribution of numerical variables; Spearman's correlation coefficients for relationships among sociodemographic variables, chronic disease, eating habits and physical activity. The study was approved by the ethics and research committees FAEN-D-912. Results: The consumption of soft drinks ( $p=.039)$, legumes $(p=.001)$ and fish $(p=.005)$ were related to schooling; water intake $(p=.007)$, legumes $(p<001)$ and schooling $(p<.001)$ were related to age; walking per week $(p<.001)$ and walking minutes $(p<.001)$ were related to water consumption. Conclusions: Diet and physical activity in older adults are important for the prevention and control of chronic diseases and other diseases. The results of this study reflect the urgency of early intervention to emphasize balanced eating habits and the regular practice of health-related physical activity.
\end{abstract}

Key words: Eating behavior; Physical activity related to health; Elderly.

\title{
RESUMO
}

Objetivo. Determinar os fatores relacionados aos hábitos alimentares e atividade física em idosos com doença crônica. Material e Método: Estudo correlacional transversal em 200 idosos de Matamoros, Tamaulipas, México. O trabalho de campo foi realizado no primeiro semestre de 2019 e por meio de um questionário deliberado foram mensurados os hábitos alimentares e a atividade física. Medidas de tendência central e dispersão foram utilizadas para variáveis contínuas; frequências e percentuais para variáveis qualitativas; teste de KolmogorovSmirnov com correção de Lilliefors para determinar a distribuição das variáveis numéricas; coeficientes de correlaçáo de Spearman para relaçóes entre variáveis sociodemográficas, doença crônica, hábitos alimentares e atividade física. O estudo foi aprovado pelos comitês de ética e pesquisa (FAEN-D-912). Resultados: O consumo de refrigerantes $(p=.039)$, legumes $(p=.001)$ e peixe $(p=.005)$ foram relacionados à escolaridade; a ingestáo de água $(p=.007)$, leguminosas $(p=.001)$ e escolaridade $(p=.001)$ foram relacionadas à idade; a caminhada por semana $(p<.001)$ e os minutos dedicados à caminhada $(p<.001)$ estavam relacionados ao consumo de água. Conclusóes: A alimentação e a atividade física em idosos são importantes para a prevenção e o controle de doenças crônicas e outros tipos de doenças. Os resultados deste estudo refletem a urgência de intervir nas fases iniciais para enfatizar os hábitos de uma alimentação equilibrada e a prática regular de atividade física associada à saúde.

Palavras-chave: Comportamento alimentar; Atividade física associada à saúde; Idoso.

Fecha de recepción: 07/04/2020

Fecha de aceptación: 30/09/2020 


\section{INTRODUCCIÓN}

Con la edad se incrementa la probabilidad de sufrir enfermedades con impacto en la salud, las relaciones sociales y economía del adulto mayor, su familia y sociedad ${ }^{(1)}$. Los hábitos alimentarios saludables y la actividad física ayudan a lo largo de la vida a prevenir o retrasar enfermedades no transmisibles y otras afecciones ${ }^{(2)}$; además, son fundamentales en todas las edades, especialmente en la población envejecida ${ }^{(3)}$.

Las enfermedades crónicas son parcial o totalmente prevenibles a través de la modificación del estilo de vida y factores de riesgo. La Organización Mundial de la Salud (OMS) plantea que los hábitos alimentarios no saludables y la inactividad física son factores de riesgo que favorecen la prevalencia de Enfermedades No Transmisibles (ENT) y muertes por comorbilidades ${ }^{(4)}$. Estos factores de riesgo pueden manifestarse en forma de hipertensión arterial, aumento de glucosa y lípidos en sangre, y acumulación de grasa corporal ${ }^{(5)}$.

Las ENT implican tratamiento de por vida y modificación del estilo de vida, incluyendo la alimentación. La composición exacta de una alimentación saludable, equilibrada y variada, dependedecada persona, considerandosuedad, sexo, hábitos de vida, ejercicio físico, contexto cultural, alimentos disponibles y hábitos alimentarios ${ }^{(6,7)}$. La OMS indica que una dieta sana incluye: frutas y verduras (cinco porciones por día), menos ingesta calórica de azucares libres (5\%) procedentes de grasas no saturadas (30\%) y menos de 5 gramos (aproximadamente una cucharadita) al día de sal yodada. De igual forma, el consumo diario de frutas y verduras puede contribuir a la prevención de enfermedades cardiovasculares y algunos cánceres, diabetes y obesidad, así como prevenir y mitigar varias carencias de micronutrientes ${ }^{(4)}$. El aumento del consumo de frutas y verduras puede ayudar a desplazar los alimentos ricos en grasas saturadas, azúcares o sal, lo que reduce el riesgo cardiovascular $^{(7)}$. Así, los hábitos alimentarios son considerados comportamientos conscientes, colectivos y repetitivos, que llevan a las personas a seleccionar, consumir y utilizar determinados alimentos o dietas, en respuesta a influencias sociales y culturales $^{(8)}$. Particularmente en los adultos mayores se ha identificado preferencia por preparaciones tradicionales y culturales, tres tiempos principales de comida, bajo consumo de lácteos, frutas y verduras y alto consumo de alimentos de elevada densidad energética ${ }^{(9)}$.

Por otro lado, la evidencia indica que los adultos mayores, hombres y mujeres con mayor actividad, presentan tasas más bajas de mortalidad y de morbilidades como cardiopatía coronaria, hipertensión, accidente cerebrovascular, diabetes tipo 2, cáncer de colon y cáncer de mama, a la vez que funciones cardiorrespiratorias y musculares mejoradas, composición corporal más sana y mejor salud ósea ${ }^{(7,8)}$. También la actividad física está asociada a mejor salud funcional, menor riesgo de caídas, mejora de las funciones cognitivas y reducción del riesgo de ENT y depresión ${ }^{(10)}$.

En este contexto, la recomendación de actividad física en el adulto mayor consiste en actividades recreativas, desplazamientos (paseos caminando o en bicicleta), actividades ocupacionales, tareas domésticas, juegos, deportes o ejercicios programados en el ámbito de las actividades diarias, familiares y comunitarias ${ }^{(5)}$. Por ello la OMS establece como estándar de oro dedicar 150 minutos semanales a diversos ejercicios ${ }^{(4)}$.

Teniendo presente lo descrito y considerando que en Tamaulipas, México, la hipertensión, diabetes y obesidad se consideran las principales causas de morbimortalidad en el adulto mayor ${ }^{(11)}$, el propósito de este estudio fue determinar los factores relacionados con los hábitos alimentarios y actividad física en adultos mayores con enfermedad crónica.

La importancia de este estudio está basada en la necesidad de conocer los hábitos alimenticios y la actividad física en este grupo, incluyendo actividades recreativas, de ocio, ocupacionales y domésticas. Con este conocimiento se espera promover hábitos de alimentación saludables y actividad física en el adulto mayor de acuerdo con sus características y necesidades.

\section{MATERIAL Y MÉTODO}

Tipo de estudio y muestreo. Se realizó un estudio descriptivo y correlacional de corte transversal, en una muestra de 200 adultos mayores de Matamoros, Tamaulipas, México. Debido a que se no se cuenta con un censo de la población adulta mayor de esta ciudad, se optó por un muestreo no probabilístico por conveniencia, bajo el criterio de ser persona 
adulta mayor y tener la capacidad de responder de manera coherente al entrevistador. Se localizaron por referencia de compańeros de trabajo y bola de nieve. A cada participante se le preguntó si recomendaba a algún vecino o conocido para participar en el estudio y de ser así, se contactó telefónicamente, se programó la cita y se siguió con el procedimiento.

Recolección de datos. Para la medición de las variables sociodemográficas se utilizó una cédula de datos personales que incluía edad, género, años de estudio y estado civil; además, si padecía alguna enfermedad, cuál y fecha de inicio de la misma. Se diseñó un cuestionario ex profeso de hábitos alimentarios que consultó sobre el número de alimentos, bebidas diarias y semanales que el adulto mayor acostumbraba a consumir. Para la medición de la actividad física se preguntó si caminaba y cuántas veces en los últimos siete días, incluyendo el reporte de minutos al día y el total semanal. Otras actividades incluidas para la medición de esta variable, que generan perdida de energía en los adultos mayores, se clasificaron en cuatro grupos: a) Actividades recreativas o de ocio: manualidades, asistir a la iglesia, jugar con nietos, jugar lotería y pintar; b) Actividades de desplazamientos: viajes/ paseos; c) Actividades ocupacionales: trabajar el jardín, llevar nińos/nietos a la escuela, realizar compras, actividades de iglesia, cuidados de nietos y cuidados de familiares y d) Actividades domésticas: barrer la calle, preparar comidas, cuidado de la casa, lavado de ropa.

Procedimiento: El trabajo de campo se realizó en el primer semestre de 2019. Se inició dando a conocer a compañeros de trabajo en la Unidad Académica Multidisciplinaria de Matamoros-UAT. Se formuló una lista con nombres, domicilios y teléfonos de adultos mayores candidatos potenciales para realizar comunicación telefónica con ellos, explicarles el estudio brevemente y solicitar una cita para visitarlos. Esta conversación se aprovechó para valorar si estaban orientados, podían escuchar y responder al entrevistador y con ello reducir sesgos. Una vez localizado el participante potencial, se le visitó en el lugar, respetando día y hora que mejor convenía a él/ella. Al inicio de la entrevista se aclaró que se le harían algunas preguntas por espacio de 30 minutos a una hora, se explicó el estudio y se obtuvo su consentimiento informado.

La entrevista fue personalizada, se les leyeron las instrucciones y las preguntas de los cuestionarios.
El entrevistador anotaba la opción de respuesta de cada participante. En todo momento el investigador principal estuvo atento a signos de cansancio, distracción y se le preguntaba si estaba cansado y deseaba continuar otro día (no se presentó esta situación). Una vez concluida la recolección de la información se agradeció a los participantes su colaboración y apoyo. Para aquellos que reportaron no tener teléfono, se hizo una primera visita para explicar el estudio, verificar criterios de inclusión y solicitar su participación. Se acordó una cita con él/ella. En el caso de que en un domicilio hubiera más de un adulto mayor, se les preguntó si ambos querían participar, siempre y cuando no escucharan lo que el otro respondía. En caso negativo se consideró al que aceptó participar.

Procesamiento y análisis de datos. Los datos se procesaron en el paquete estadístico Statistical Package for Social Siences (SPSS) versión 22.0 para Windows. Se usaron medidas de tendencia central y de dispersión para describir las variables continuas; frecuencias y porcentajes para las variables cualitativas. Se aplicó la prueba de KolmogorovSmirnov con corrección de Lilliefors para determinar la distribución de variables numéricas y decidir el uso de estadística paramétrica o no paramétrica. Se usaron coeficientes de correlación de Spearman para las relaciones entre las variables sociodemográficas, enfermedad crónica, hábitos alimenticios y actividad física.

Consideraciones éticas. La investigación consideró el reglamento de la Ley General de Salud en Materia de Investigación para la salud, de México $^{(12)}$ en sus artículos 13, 14, 16-18, 20-22, relacionados con la dignidad de los participantes, consentimiento informado por escrito, privacidad de los participantes, investigación calificada sin riesgo y la libertad de negarse a seguir participando en el estudio en el momento que así lo decidieran. El estudio fue aprobado por los comités de ética e investigación de la Facultad de Enfermería de la UANL (FAEN-D-912).

\section{RESULTADOS}

Caracterización de los adultos mayores. El grupo de adultos mayores que participaron del estudio tenía una media de edad de 68,98 años (DE= 7,60) con valores entre los 60 y 91 años $K-S$ (105), en 
su mayoría eran mujeres $(64,5 \%)$, tenían pareja $(54,5 \%)$ y una media de escolaridad de 10,21 años $(\mathrm{DE}=6,63)$. La enfermedad crónica más prevalente fue Hipertensión $(43,5 \%)$ y el $13,5 \%$ presentó otras enfermedades que fueron reportadas como cáncer de mama, osteoartrosis, cardiovasculares y problemas prostáticos. El rango de años que padecían la enfermedad osciló entre 1 hasta 37 y la mayoría (61\%) se reportó en el grupo que tenía entre 15 o menos ańos de padecerla.

Hábitos alimentarios de los adultos mayores. En el caso de frutas por día la media fue $1,53(\mathrm{DE}=, 820)$, verduras por día $\mathrm{X}=1,55(\mathrm{DE}=, 819)$, legumbres por día $\mathrm{X}=3,82(\mathrm{DE}=1,513)$, pollo por semana $\mathrm{X}=$ $2,54(D E=1,079)$, postres-dulces por día $\mathrm{X}=2,39$ $(D E=1,352)$, vasos de refresco por día $\mathrm{X}=1,77$ $(D E=1,340)$ y vasos de agua por día $\mathrm{X}=4,37(D E=$ 1,521). Tanto las frutas como las verduras al día son los alimentos menos consumidos con valores desde 0 a 5 , y en el caso del refresco y el agua, son los más consumidos con valores de 0 a 8 y 1 a 8 vasos al día, respectivamente. En la Tabla 1 se puede observar que el 90,5\% (181) de los participantes, señaló consumir de 1 a 3 frutas diarias, el 93\% (186) de 1-3 verduras, el 28\% (56) de 1-3 vasos de agua, el 76,5\% (153) refirió consumir de 1-3 vasos de refresco o agua endulzada. Además, se realizó un análisis de datos cruzados entre el consumo de alimentos diarios con variables como género, estado marital, escolaridad y años con enfermedad, donde se encontró una relación entre el consumo de refrescos con la escolaridad $\left(\mathrm{X}^{2}=6,48 ; p=, 039\right)$.

Relación entre variables sociodemográficas con hábitos de consumo de alimentos por semana (Tabla 2). Se realizó un análisis de tablas cruzadas para las mismas variables sociodemográficas y de años con la enfermedad crónica y la frecuencia de algunos grupos de alimentos que se consumen por semana, en donde se encontró que el 55\% (110) refieren comer legumbres más de 4 veces a la semana; además se encontró una relación entre la frecuencia de este consumo de alimento con la escolaridad $\left(\mathrm{X}^{2}=13,50 ; p=, 001\right)$ y con los años de enfermedad $\left(\mathrm{X}^{2}=7,41 ; p=, 025\right)$. El 83,5\% (167) de los participantes consume pollo de 1-3 días a la semana y el 66\% (132) consume pescado con la misma frecuencia; además esto se asoció con la escolaridad $\left(\mathrm{X}^{2}=7,85 ; p=, 005\right)$ y con los años de enfermedad $\left(X^{2}=17,01 ; p=, 001\right)$. Por otro lado, el 67,5\% (135) mencionó que ingieren postres o dulces 1-3 veces por semana, lo cual se relacionó con el estado marital $\left(\mathrm{X}^{2}=10,37 ; p=, 006\right)$ y con la escolaridad $\left(\mathrm{X}^{2}=10,23 ; p=, 006\right)$.

Actividad física según variables sociodemográficas de adultos mayores (Tabla 3). De acuerdo con las actividades domésticas por día, la actividad física mayormente reportada fue la limpieza de la casa $(f=175)$ que representó el $32,8 \%$ del total de las actividades diarias y de acuerdo con las variables sociodemográficas, se concentró mayor frecuencia de esta actividad en las participantes de género femenino, con pareja, menos años de escolaridad y menos años con enfermedad. En relación con otras actividades de desplazamiento por semana, ir de compras es la actividad más realizada $(f=175)$ que representa el 95,6\% del total de actividades por desplazamiento. Respecto a caminar ( $f=$ 78) mencionaron que caminan de 1-4 días por semana, lo que representa el $39 \%$ de esta actividad semanal, y más de 150 minutos acumulados a la semana $(f=10)$ que representa solamente el $5 \%$ de esta actividad. Cabe mencionar que respecto a otras actividades reportadas por los participantes (bicicleta fija, caminadora, GYM, nado), la realizaban solamente 45 participantes, de los cuales 25 eran de sexo femenino y 20 de sexo masculino.

Correlación entre variables sociodemográficas, de la enfermedad crónica y actividad física con hábitos alimentarios de adultos mayores. Finalmente, se realizó un análisis de correlación con el estadístico Spearman para variables sin distribución normal (Tabla 4) y se encontró una relación entre la edad y la ingesta de vasos de agua al día $\left(r_{s}=\right.$ -,189; $p=, 007)$. Los años de estudio se relacionaron con la misma variable $\left(r_{s}=, 250 ; p<, 001\right)$ y con el consumo de legumbres por semana $\left(r_{s}=-, 320 ; p<\right.$ ,001). Así mismo, esta variable se relacionó con los años con la enfermedad $\left(r_{s}=, 165 ; p=, 019\right)$ y a su vez esta última con el consumo de vasos de agua al día $\left(r_{s}=-, 259 ; p^{<}, 001\right)$. Por último, respecto a la relación entre hábitos alimentarios y actividad física, se encontró que los días a la semana que los participantes caminan se relaciona con el consumo de vasos de agua al día $\left(r_{s}=, 249 ; p<, 001\right)$. Así mismo, el consumo de vasos de agua se relaciona con los minutos dedicados a caminar por semana $\left(r_{s}=, 290 ; p<, 001\right)$ y a su vez con las raciones de fruta diarias $\left(r_{s}=, 156 ; p=, 027\right)$. 
Tabla 1a. Distribución frecuencial, porcentual y relacional entre consumo de alimentos y variables sociodemográficas de adultos mayores, Tamaulipas, México, 2019 ( $\mathrm{n}=200)$.

\begin{tabular}{|c|c|c|c|c|c|c|c|c|c|c|c|c|c|}
\hline \multirow[t]{3}{*}{ Alimento } & \multirow{2}{*}{$\begin{array}{c}\text { Consumo } \\
\text { diario }\end{array}$} & \multicolumn{2}{|c|}{ Género } & \multicolumn{10}{|c|}{ Estado marital } \\
\hline & & Femenino & Masculino & Total & $\%$ & $\mathrm{X}^{2}$ & $\mathrm{p}$ & C/pareja & S/pareja & Total & $\%$ & $\mathrm{X}^{2}$ & $\mathrm{p}$ \\
\hline & & $f$ & $f$ & & & & & $f$ & $f$ & & & & \\
\hline \multirow{4}{*}{ Frutas } & 0 por día & 9 & 6 & 15 & 7,5 & 0,327 & 0,849 & 6 & 9 & 15 & 7,5 & 6,486 & 0,039 \\
\hline & 1-3 diarias & 117 & 64 & 181 & 90,5 & & & 103 & 78 & 181 & 90,5 & & \\
\hline & $>4$ diarias & 3 & 1 & 4 & 2,0 & & & 0 & 4 & 4 & 2,0 & & \\
\hline & Total & 129 & 71 & 200 & 100,0 & & & 109 & 91 & 200 & 100,0 & & \\
\hline \multirow{4}{*}{ Verduras } & 0 por día & 7 & 4 & 11 & 5,5 & 0,01 & 0,995 & 8 & 3 & 11 & 5,5 & 2,056 & 0,358 \\
\hline & 1-3 diaria & 120 & 66 & 186 & 93,0 & & & 100 & 86 & 186 & 93,0 & & \\
\hline & $>4$ diarias & 2 & 1 & 3 & 1,5 & & & 1 & 2 & 3 & 1,5 & & \\
\hline & Total & 129 & 71 & 200 & 100,0 & & & 109 & 91 & 200 & 100,0 & & \\
\hline \multirow{4}{*}{$\begin{array}{l}\text { Vasos de } \\
\text { agua }\end{array}$} & 0 por día & 0 & 0 & 0 & 0,0 & 1,054 & 0,304 & 0 & 0 & 0 & 0,0 & 1,211 & 0,271 \\
\hline & $1-3$ veces & 33 & 23 & 56 & 28,0 & & & 34 & 22 & 56 & 28,0 & & \\
\hline & $>4$ veces & 96 & 48 & 144 & 72,0 & & & 75 & 69 & 144 & 72,0 & & \\
\hline & Total & 129 & 71 & 200 & 100,0 & & & 109 & 91 & 200 & 100,0 & & \\
\hline \multirow{4}{*}{$\begin{array}{l}\text { Vasos de } \\
\text { refresco } \\
\text { o agua } \\
\text { endulzada }\end{array}$} & 0 veces & 17 & 12 & 29 & 14,5 & 0,524 & 0,769 & 14 & 15 & 29 & 14,5 & 1,531 & 0,465 \\
\hline & $1-3$ veces & 100 & 53 & 153 & 76,5 & & & 87 & 66 & 153 & 76,5 & & \\
\hline & $>4$ veces & 12 & 6 & 18 & 9,0 & & & 8 & 10 & 18 & 9,0 & & \\
\hline & Total & 129 & 71 & 200 & 100,0 & & & 109 & 91 & 200 & 100,0 & & \\
\hline
\end{tabular}

Nota: $\mathrm{p}=$ significancia estadística; $\mathrm{X}^{2}=$ Chi Cuadrada; ${ }^{*}=(\mathrm{p}<, 05)$

Tabla 1 b.

\begin{tabular}{|c|c|c|c|c|c|c|c|c|c|c|c|c|c|}
\hline \multirow[t]{3}{*}{ Alimento } & \multirow{3}{*}{$\begin{array}{c}\text { Consumo } \\
\text { diario }\end{array}$} & \multicolumn{2}{|c|}{ Escolaridad } & \multicolumn{10}{|c|}{ Ańos con Enfermedad } \\
\hline & & $\leq 14$ años & $\geq 15$ años & \multirow[t]{2}{*}{ Total } & \multirow[t]{2}{*}{$\%$} & \multirow[t]{2}{*}{$\mathrm{X}^{2}$} & \multirow[t]{2}{*}{$\mathrm{p}$} & $\leq 14$ & $\geq 15$ & \multirow[t]{2}{*}{ Total } & \multirow[t]{2}{*}{$\%$} & \multirow[t]{2}{*}{$\mathrm{X}^{2}$} & \multirow[t]{2}{*}{$\mathrm{p}$} \\
\hline & & $f$ & $f$ & & & & & $f$ & $f$ & & & & \\
\hline \multirow{4}{*}{ Frutas } & 0 por día & 9 & 6 & 15 & 7,5 & 2,324 & 0,313 & 10 & 5 & 15 & 7,5 & 2,895 & 0,235 \\
\hline & 1-3 diarias & 123 & 58 & 181 & 90,5 & & & 108 & 73 & 181 & 90,5 & & \\
\hline & $>4$ diarias & 4 & 0 & 4 & 2 & & & 4 & 0 & 4 & 2 & & \\
\hline & Total & 136 & 64 & 200 & 100 & & & 122 & 78 & 200 & 100 & & \\
\hline \multirow{4}{*}{ Verduras } & 0 por día & 6 & 5 & 11 & 5,5 & 2,334 & 0,311 & 8 & 3 & 11 & 5,5 & 2,691 & 0,26 \\
\hline & 1-3 diaria & 127 & 59 & 186 & 93 & & & 111 & 75 & 186 & 93 & & \\
\hline & $>4$ diarias & 3 & 0 & 3 & 1,5 & & & 3 & 0 & 3 & 1,5 & & \\
\hline & Total & 136 & 64 & 200 & 100 & & & 122 & 78 & 200 & 100 & & \\
\hline \multirow{4}{*}{$\begin{array}{l}\text { Vasos de } \\
\text { agua }\end{array}$} & 0 por día & 0 & 0 & 0 & 0 & 1,751 & 0,186 & 0 & 0 & 0 & 0 & 1,804 & 0,179 \\
\hline & $1-3$ veces & 42 & 14 & 56 & 28 & & & 30 & 26 & 56 & 28 & & \\
\hline & $>4$ veces & 94 & 50 & 144 & 72 & & & 92 & 52 & 144 & 72 & & \\
\hline & Total & 136 & 64 & 200 & 100 & & & 122 & 78 & 200 & 100 & & \\
\hline \multirow{4}{*}{$\begin{array}{l}\text { Vasos de } \\
\text { refresco } \\
\text { o agua } \\
\text { endulzada }\end{array}$} & 0 veces & 25 & 4 & 29 & 14,5 & 7,223 & 0,027 & 20 & 9 & 29 & 14,5 & 5,648 & 0,059 \\
\hline & $1-3$ veces & 102 & 51 & 153 & 76,5 & & & 87 & 66 & 153 & 76,5 & & \\
\hline & $>4$ veces & 9 & 9 & 18 & 9 & & & 15 & 3 & 18 & 9 & & \\
\hline & Total & *136 & $* 64$ & 200 & 100 & & & 122 & 78 & 200 & 100 & & \\
\hline
\end{tabular}


Tabla 2a. Relación entre variables sociodemográficas con hábitos de consumo de alimentos por semana de adultos mayores, Tamaulipas, México, 2019 ( $\mathrm{n}=200)$.

\begin{tabular}{|c|c|c|c|c|c|c|c|c|c|c|}
\hline \multirow[t]{3}{*}{ Alimento } & \multirow{3}{*}{$\begin{array}{c}\text { Consumo } \\
\text { semanal }\end{array}$} & \multicolumn{2}{|c|}{ Género } & \multirow{3}{*}{ Total } & \multirow{3}{*}{$\%$} & \multirow{3}{*}{$\mathrm{X}^{2}$} & \multicolumn{4}{|c|}{ Estado marital } \\
\hline & & Femenino & Masculino & & & & $\mathrm{p}$ & Con pareja & Sin pareja & Total \\
\hline & & $f$ & $f$ & & & & & $f$ & $f$ & \\
\hline \multirow{4}{*}{ Legumbres } & 0 veces & 0 & 2 & 2 & 1,0 & 3,829 & 0,147 & 2 & 0 & 2 \\
\hline & 1-3 veces & 56 & 32 & 88 & 44,0 & & & 49 & 39 & 88 \\
\hline & $>4$ veces & 73 & 37 & 110 & 55,0 & & & 58 & 52 & 110 \\
\hline & Total & 129 & 71 & 200 & 100,0 & & & 109 & 91 & 200 \\
\hline \multirow{4}{*}{ Pollo } & 0 veces & 2 & 2 & 4 & 2,0 & 0,633 & 0,729 & 4 & 0 & 4 \\
\hline & $1-3$ veces & 107 & 60 & 167 & 83,5 & & & 90 & 77 & 167 \\
\hline & $>4$ veces & 20 & 9 & 29 & 14,5 & & & 15 & 14 & 29 \\
\hline & Total & 129 & 71 & 200 & 100,0 & & & 109 & 91 & 200 \\
\hline \multirow{4}{*}{ Pescado } & 0 veces & 41 & 27 & 68 & 34,0 & 0,796 & 0,372 & 33 & 35 & 68 \\
\hline & 1-3 veces & 88 & 44 & 132 & 66,0 & & & 76 & 56 & 132 \\
\hline & $>4$ veces & 0 & 0 & 0 & 0,0 & & & 0 & 0 & 0 \\
\hline & Total & 129 & 71 & 200 & 100,0 & & & 109 & 91 & 200 \\
\hline \multirow{4}{*}{$\begin{array}{l}\text { Postres, } \\
\text { Dulces, Bollos }\end{array}$} & 0 veces & 14 & 5 & 19 & 9,5 & 0,78 & 0,677 & 5 & 14 & 19 \\
\hline & $1-3$ veces & 86 & 49 & 135 & 67,5 & & & 72 & 63 & 135 \\
\hline & $>4$ veces & 29 & 17 & 46 & 23,0 & & & 32 & 14 & 46 \\
\hline & Total & 129 & 71 & 200 & 100,0 & & & **109 & ${ }^{* *} 91$ & 200 \\
\hline
\end{tabular}

Nota: $\mathrm{p}=$ significancia estadística; $\mathrm{X}^{2}=$ Chi Cuadrada; ${ }^{*}=(\mathrm{p}<, 05) ;{ }^{* *}=(\mathrm{p}<, 01)$

Tabla 2 b.

\begin{tabular}{|c|c|c|c|c|c|c|c|c|c|c|c|c|c|}
\hline \multirow[t]{3}{*}{ Alimento } & \multirow{3}{*}{$\begin{array}{c}\text { Consumo } \\
\text { semanal }\end{array}$} & \multicolumn{3}{|c|}{ Escolaridad } & \multicolumn{9}{|c|}{ Años con Enfermedad } \\
\hline & & $\leq 14$ años & $\geq 15$ ańos & Total & $\%$ & $\mathrm{X}^{2}$ & $\mathrm{p}$ & $\leq 14$ ańos & $\geq 15$ años & Total & $\%$ & $\mathrm{X}^{2}$ & $\mathrm{p}$ \\
\hline & & $f$ & $f$ & & & & & $f$ & $f$ & & & & \\
\hline \multirow{4}{*}{ Legumbres } & 0 veces & 2 & 0 & 2 & 1,0 & 13,503 & 0,001 & 1 & 1 & 2 & 1 & 7,415 & 0,025 \\
\hline & $1-3$ veces & 48 & 40 & 88 & 44,0 & & & 63 & 25 & 88 & 44 & & \\
\hline & $>4$ veces & 86 & 24 & 110 & 55,0 & & & 58 & 52 & 110 & 55 & & \\
\hline & Total & **136 & $* * 64$ & 200 & 100,0 & & & $* 122$ & $* 78$ & 200 & 100 & & \\
\hline \multirow{4}{*}{ Pollo } & 0 veces & 2 & 2 & 4 & 2,0 & 0,864 & 0,649 & 4 & 0 & 4 & 2 & 3,638 & 0,162 \\
\hline & $1-3$ veces & 113 & 54 & 167 & 83,5 & & & 103 & 64 & 167 & 83,5 & & \\
\hline & $>4$ veces & 21 & 8 & 29 & 14,5 & & & 15 & 14 & 29 & 14,5 & & \\
\hline & Total & 136 & 64 & 200 & 100,0 & & & 122 & 78 & 200 & 100 & & \\
\hline \multirow{4}{*}{ Pescado } & 0 veces & 55 & 13 & 68 & 34,0 & 7,858 & 0,005 & 28 & 40 & 68 & 34 & 17,019 & 0,001 \\
\hline & $1-3$ veces & 81 & 51 & 132 & 66,0 & & & 94 & 38 & 132 & 66 & & \\
\hline & $>4$ veces & 0 & 0 & 0 & 0,0 & & & 0 & 0 & 0 & 0 & & \\
\hline & Total & ${ }^{* *} 136$ & $* * 64$ & 200 & 100,0 & & & ${ }^{* *} 122$ & $* * 78$ & 200 & 100 & & \\
\hline \multirow{4}{*}{$\begin{array}{l}\text { Postres, } \\
\text { Dulces, Bollos }\end{array}$} & 0 veces & 18 & 1 & 19 & 9,5 & 10,231 & 0,006 & 10 & 9 & 19 & 9,5 & 5,852 & 0,054 \\
\hline & $1-3$ veces & 93 & 42 & 135 & 67,5 & & & 77 & 58 & 135 & 67,5 & & \\
\hline & $>4$ veces & 25 & 21 & 46 & 23,0 & & & 35 & 11 & 46 & 23 & & \\
\hline & Total & **136 & $* * 64$ & 200 & 100,0 & & & 122 & 78 & 200 & 100 & & \\
\hline
\end{tabular}


Tabla 3a. Distribución frecuencial y porcentual de la actividad física según variables sociodemográficas de adultos mayores, Tamaulipas, México, 2019 ( $\mathrm{n}=200)$.

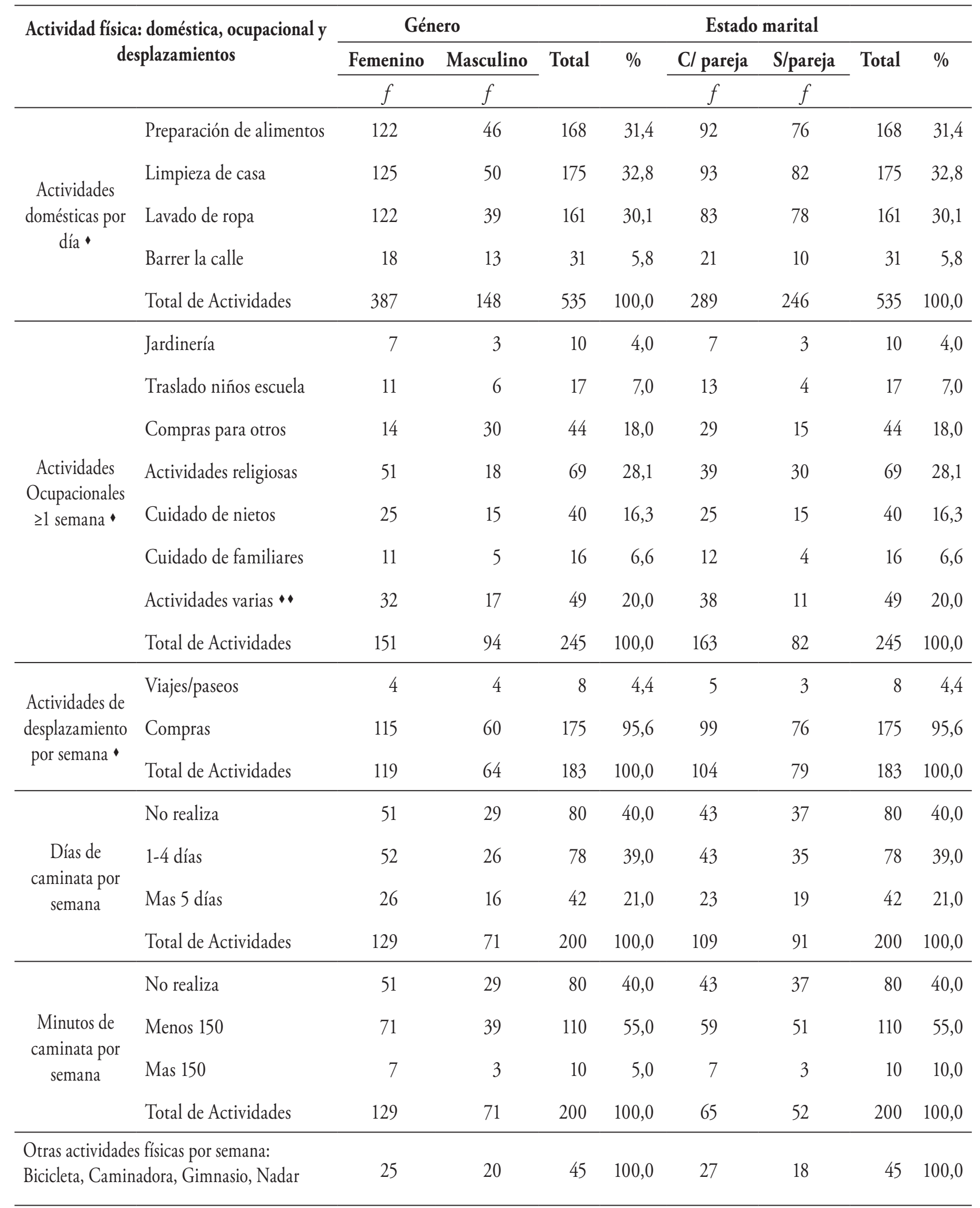

- Cada adulto mayor tuvo la posibilidad de indicar más de una opción de respuesta.

- Comunitarias, educativas, culturales y beneficencia. 
Tabla 3b. Distribución frecuencial y porcentual de la actividad física según variables sociodemográficas de adultos mayores, Tamaulipas, México, 2019 ( $\mathrm{n}=200)$.

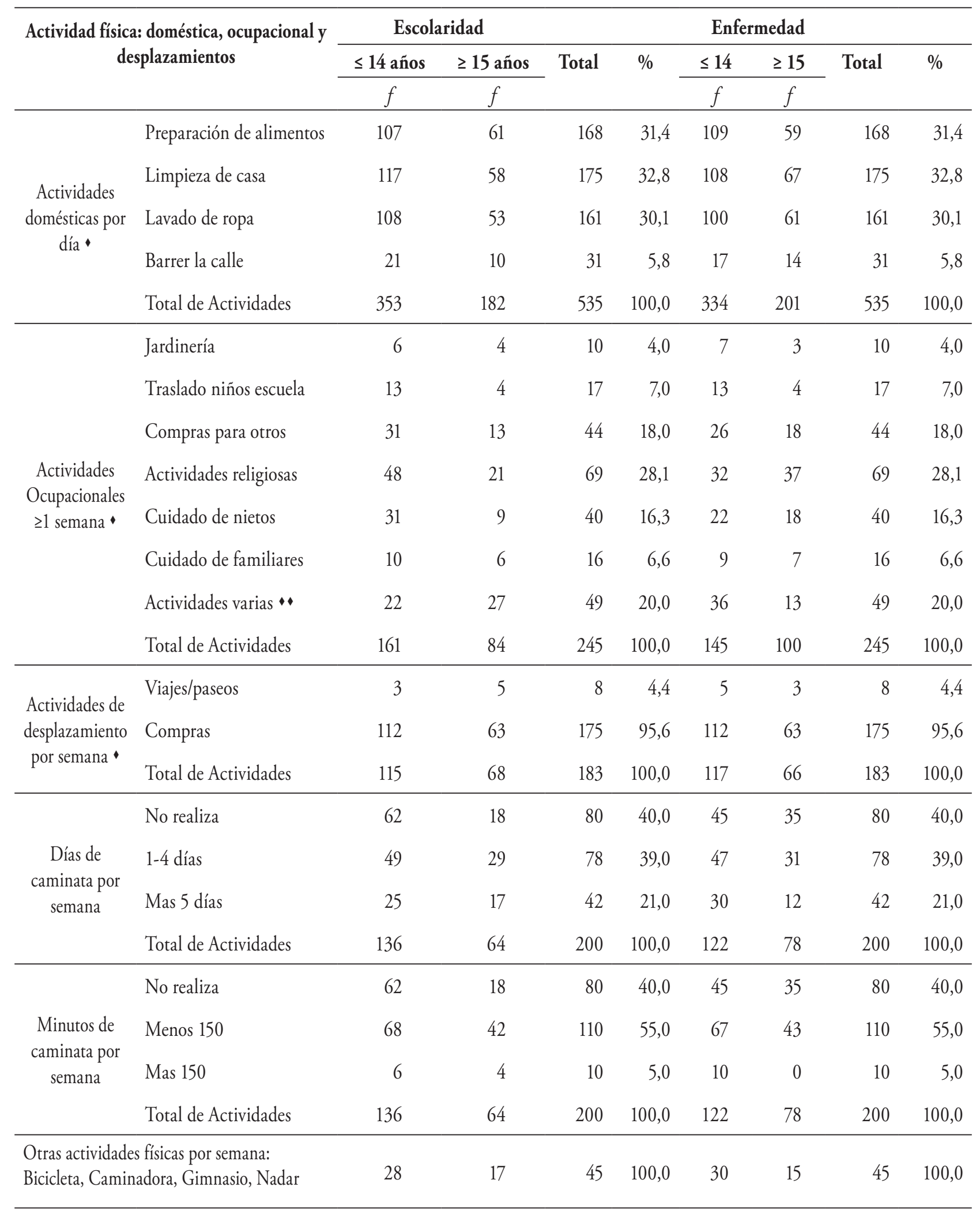

- Cada adulto mayor tuvo la posibilidad de indicar más de una opción de respuesta.

- Comunitarias, educativas, culturales y beneficencia. 
Tabla 4. Correlación entre variables sociodemográficas, de la enfermedad crónica y actividad física con hábitos alimentarios de adultos mayores, Tamaulipas, México, 2019 ( $\mathrm{n}=200)$.

\begin{tabular}{lccccc}
\hline \multirow{2}{*}{ Hábitos Alimentarios } & Edad & $\begin{array}{c}\text { Años de } \\
\text { estudio }\end{array}$ & $\begin{array}{c}\text { Años con } \\
\text { enfermedad }\end{array}$ & $\begin{array}{c}\text { Días a la } \\
\text { semana que } \\
\text { camina }\end{array}$ & $\begin{array}{c}\text { Minutos que } \\
\text { camina a la } \\
\text { semana }\end{array}$ \\
\cline { 2 - 6 } & rs & rs & rs & rs & rs \\
\hline Raciones de frutas $^{\mathrm{a}}$ & $-0,088$ & 0,108 & $-0,117$ & 0,107 & $.156^{*}$ \\
Raciones de verduras $^{\mathrm{a}}$ & $-0,019$ & $-0,072$ & $-0,114$ & $-0,006$ & 0,021 \\
Legumbres $^{\mathrm{b}}$ & 0,072 & $-.320^{* *}$ & $.165^{*}$ & $-0,132$ & $-0,097$ \\
Pollo $^{\mathrm{b}}$ & 0,002 & 0,036 & $-0,046$ & 0,042 & 0,075 \\
Vasos de agua al día & $-.189^{* *}$ & $.250^{* *}$ & $-.259^{* *}$ & $.249^{* *}$ & $.290^{* *}$ \\
\hline $\mathrm{rs}=$ Coeficiente de Spearman;
\end{tabular}

\section{DISCUSIÓN}

En el presente estudio se logró describir los hábitos alimentarios y actividad física de los adultos mayores, así como la relación entre estas variables. Se evidenció empíricamente que el consumo de refrescos, legumbres y pescado se relacionaron con la escolaridad, y la ingesta de agua y legumbres, así como la escolaridad está relacionada con la edad. La caminata por semana, y el tiempo que dedican a caminar los adultos mayores se relaciona con el consumo de agua; las raciones de fruta que consume el adulto mayor se relacionaron con el tiempo en minutos que dedica a la caminata por semana.

El mayor consumo de frutas y verduras reportado por los adultos mayores es de 1-3 piezas y se encuentra por sobre el $90 \%$, sin embargo, la recomendacion para estos alimentos, según la OMS, es de $400 \mathrm{~g}$ diarios $^{(13)}$. En este trabajo se preguntó exclusivamente por el número de frutas y verdura consumidas y para aquellos que reportaron una pieza diaria o una porción de verdura diaria no se cumple con el estándar propuesto. El hecho de consultar por número de piezas o unidades consumidas es una forma más comprensible para responder por el adulto mayor.

A nivel mundial se encontró un resultado que menciona un déficit del $87 \%$ en consumo de frutas y verduras ${ }^{(14)}$. Por otro lado, en México Ramírez-Silva, Rivera, Ponce y Hernández-Ávila reportaron resultados obtenidos de la Encuesta Nacional de Salud y Nutrición en el 2006, donde solo el 30\% de la población en México tenía un consumo adecuado de frutas y verduras, datos muy por arriba a lo encontrado en este estudio, donde la media de frutas y verduras es de $1,53^{(15)}$. Así mismo, el programa $5 \mathrm{x}$ día señala 5 porciones de frutas y/o verduras al día, pero en este trabajo solo el $10 \%$ de la muestra cumplió con el estándar de dicho programa ${ }^{(16)}$. Ante estos resultados se sugiere promocionar y dar a conocer los beneficios protectores del consumo de frutas y verduras relacionados con enfermedades vasculares y cáncer.

El consumo de legumbres más conocido en la región son los frijoles y lentejas y la Organización de las Naciones Unidas para la Alimentación (FAO) y la OMS, recomiendan un consumo de $400 \mathrm{~g}$ al día o más de 3 veces por semana ${ }^{(17)}$. Los resultados en este apartado indican que el 55\% señaló consumir más de 4 veces por semana y una media de 3,82, datos que son compatibles con lo recomendado. Aunado a esto, en los últimos años, los reportes de la FAO mencionan que el consumo de legumbres ha bajado de aproximadamente $10 \mathrm{~kg}$ por persona a poco más de $6 \mathrm{~kg}$ por persona al año. Las legumbres sin duda representan una buena opción para sustituir el aporte de proteínas de origen animal y además de ser más económicas, en el caso de los adultos mayores facilitan la masticación y el tránsito intestinal ${ }^{(18)}$. En México las legumbres son parte tradicional del platillo diario, por lo que, posiblemente, la disminución del consumo de este alimento se relacione con la cuestión económica o con aquellos que no tenían pareja, estos últimos representados por un poco menos de la mitad de los adultos mayores de la muestra.

Los resultados encontrados muestran relación 
entre la frecuencia de consumo de legumbres y pollo con la escolaridad, posiblemente a mayor escolaridad mejor ingreso económico. Al igual que en nuestro estudio, resultados de GaonaPineda et al. ${ }^{(19)}$, en México, reportaron que el nivel socioeconómico está relacionado con el ingreso y probablemente mayor acceso a los alimentos y la calidad en la dieta. Estas mismas variables también mostraron relación con los años de enfermedad, es decir, a mayores años con la enfermedad mayor consumo de legumbres y pollo.

El pollo lo consumen, aproximadamente, el $83,5 \%$ de los adultos mayores, con una frecuencia de 1-3 veces por semana; menos frecuente es el consumo de pescado que se representó en el 63\% y 1-3 veces por semana. Aun cuando los beneficios del consumo de carne blanca están disponibles para todo público, no se encontraron estudios del consumo de pescado ni de su frecuencia en México. Para poder comparar, se encontró un estudio de hábitos alimenticios de origen chileno, que señala que nuestro resultado es inferior a lo encontrado en ese país, cuyo consumo de pescado fue en el $90 \%$ de la muestra estudiada con una regularidad semanal ${ }^{(20)}$. Una limitante de este trabajo es no haber preguntado sobre el consumo de carne de res/puerco, ya que en México una creencia es que este tipo de carnes da mayor fortaleza para trabajar, al contrario de la carne blanca que es considerada ligera y es mejor para los niños.

Casi la tercera parte señaló tomar 4 vasos de agua natural al día. Al respecto se ha reportado una mínima reducción de enfermedad cardiovascular en los que toman cinco o más vasos de agua diarios ${ }^{(21)}$ y se ha encontrado asociación entre la ingesta total de líquidos y el riesgo de cáncer ${ }^{(22)}$. También se señala que las recomendaciones del consumo de agua diferente a otros líquidos, no son concluyentes $y$ es diferente en cada persona ${ }^{(23)}$. Continuando con el consumo de líquidos, el 10\% consume 4 vasos o más de refresco, o bebidas endulzadas, además de los azucares en los postres o bollos reportados, los cuales de 1-3 veces por semana representa el $67,5 \%$ de consumo en los participantes. Al respecto, la recomendación de la OMS señala que debe ser menor del 10\% de la ingesta calórica total de azúcares. Por otro lado, el mayor consumo de postres y dulces se relacionó con el estado marital, posiblemente debido a la tradición de las comidas mexicanas, pues el postre después de la comida es frecuente en la mayoría de los casos. Los hallazgos de este trabajo, no solo permiten conocer los hábitos del adulto mayor en relación a las bebidas, sino de toda la familia, ya que, posiblemente, en el hogar es donde se preparan las bebidas o aguas endulzadas, y pone de manifiesto la necesidad de intervenciones en la familia.

La actividad recreativa o de ocio es parte del estilo de vida para muchas personas, les mantiene activos, mejoran las relaciones sociales y contribuyen a elevar la autoestima. En este estudio fue escasa la participación en las actividades propuestas pues solo el 23,5\% de los participantes señaló tener participación en ellas. Lo que más resalta es la convivencia con nietos, aun cuando no se preguntó si la convivencia era diferente a proporcionar cuidados. Al respecto la literatura reporta efectos beneficiosos para la salud, para aquellos abuelos que cuidan nietos de forma ocasional $^{(24)}$. Posiblemente en el caso de la mujer, las abuelas pueden convertirse en cuidadoras sin que ellas mismas estén conscientes de ello por el alto grado de sentimentalismo que tiene esta tarea, aun cuando para ellas pueda ser una obligación.

Llama la atención que viajes y paseos de los adultos mayores casi no fueron reportados, a diferencia de ir de compras $(87,5 \% ; f=175)$, en comparación con la encuesta del uso del tiempo en el 2012 25 , en donde esta misma actividad se reportó en un 2,6\%. En este trabajo no se preguntó si el lugar de las compras era un supermercado, u otros establecimientos, además si las compras eran en el mismo municipio, ya que al tratarse de una ciudad fronteriza, el cruce a USA, probablemente, para algunos adultos mayores significa más que un paseo.

Otro dato interesante de los participantes fue la actividad religiosa practicada en el 34,5\% de la muestra. Se pudo observar que quienes señalaron tener actividad eran los adultos con pareja, menos escolaridad y más años de enfermedad. La adherencia a una religión ayuda a las personas a mantenerse activas y participar en las actividades. Se han reportado resultados que señalan que participar en dichas actividades es beneficioso para el cuidado de la vida y la salud y les permite, a su vez, relaciones de convivencia social ${ }^{(26)}$. Cabe destacar que las actividades de convivencia social resultaron ser más frecuentes en mujeres $(72,5 \%)$ que en hombres $(64,2 \%)$. 
Las actividades domésticas como la limpieza de la casa, la preparación de alimentos y lavado de ropa, fueron reportadas por la mayoría de los participantes. Como en otros trabajos ${ }^{(25)}$, quien realiza mayormente estas tareas es la mujer y se pudo observar que quienes reportaron con mayor frecuencia estas tareas, son los que tienen pareja, menos escolaridad y menos ańos con enfermedad. Estos resultados permiten comprobar que, posiblemente, aquellos que reportaron con menos frecuencia estas actividades son los mismos que tampoco realizan otras actividades físicas por algún grado de dependencia. Las recomendaciones de la OMS es caminar 5 o más días por 45 minutos, o una sumatoria de 150 minutos semanales, criterio que no se cumplió en los participantes, ya que el reporte total de caminar 5 o más días fue muy escaso, y un poco más, pero tampoco suficiente, fueron aquellos que señalaron caminar de 1 a 4 días por semana. De igual manera, la frecuencia de quienes realizan deporte y ejercicio físico es escasa, con predominio mayor en hombres que en mujeres, 19 y 13\%, respectivamente. La recomendación es insistir a la familia y a los adultos mayores en apoyar y atender a esta recomendación; la evidencia demuestra que la actividad a esta edad representa mayor salud funcional, menor riesgo de caídas y disminución en las tasas de mortalidad.

\section{REFERENCIAS}

1. Espinoza OL, Rodríguez RF, Gálvez CJ, MacMillan KN. Hábitos de alimentación y actividad física en estudiantes universitarios. Rev chil nutr [Internet]. 2011 [citado 9 ene 2020]; 8(4): 458-465. Disponible en: http://dx.doi. org/10.4067/S0717-75182011000400009

2. Amador LV, Ibáńez E. Calidad de vida y formación en hábitos saludables en la alimentación de personas mayores. Rev de Humanidades [Internet]. 2015 [citado 11 ene 2020]; 25: 145-168. Disponible en: http://revistas.uned.es/index.php/ $\mathrm{rdh} /$ article/view/14374

3. Instituto Nacional de Estadística y Geografía. Encuesta Nacional de la dinámica demográfica

\section{CONCLUSIONES}

Los resultados de este estudio indican que a mayor tiempo de caminata por semana, mayor consumo de porciones de fruta y vasos de agua en los adultos mayores. De manera general, los resultados muestran que los años de enfermedad juegan un papel muy importante y quienes reportan tener menos años de enfermedad tienen mayor frecuencia en el desempeño de las actividades físicas, así como mayor consumo de frutas y verduras. Las frecuencias de actividad física son mayores en las mujeres que en los hombres, excepto para los días de caminata y minutos de caminata semanal. Se puede observar que el hecho de tener pareja es sinónimo de mayor actividad. Quienes reportaron menos ańos de escolaridad en las actividades abordadas son los que mayormente participan.

Pese a que los resultados de esta investigación son de aplicación para esta población y parten de un muestreo no probabilístico por conveniencia, se considera que es un importante acercamiento a esta problemática; ya que se evidenció que la alimentación y la actividad física en los adultos mayores son importantes para la prevención y control de las enfermedades crónicas y otro tipo de enfermedades. Los resultados de este estudio reflejan la urgencia de intervenir en etapas tempranas, enfatizando en la alimentación balanceada y práctica regular de actividad física asociada a la salud.

[Internet]. 2014 [citado 20 ene 2020]. Disponible en: http://www3.inegi.org/mx/sistemas/temas/ default $/$. aspx? s=est $\& c=17484$.

4. Organización Mundial de la Salud. Estrategia mundial sobre régimen alimentario, actividad física y salud. La actividad física en los adultos mayores [Internet]. 2018. [citado 25 ene 2020]. Disponible en: www.who.int/dietphysicalactivity/ factsheet_olderadults/es/.

5. Organización Mundial de la Salud. Nueva edición sobre el informe de seguimiento de los progresos en relación con las enfermedades no transmisibles. Comunicado de prensa [Internet]. 2017 [citado 20 ene 2020]. Disponible en: https://www.who.int/es/ news-room/detail/18-09-2017-who-launches-newncds-progress-monitor 
6. Organización de las Naciones Unidas para la Alimentación y Agricultura. Recomendaciones fundamentales para mejorar la nutrición a través de la agricultura [Internet]. 2015 [citado 5 ene 2020]. Disponible en: http://www.fao.org/ documents/card/en/c/da6aa60d-6088-4501-bf4ff07d4fa6108e/

7. Organización Mundial de la Salud. Alimentación sana [Internet]. 2015 [citado 10 ene 2020]; Nota Descriptiva no 394. Disponible en: https://www. who.int/es/news-room/fact-sheets/detail/healthydiet

8. Troncoso-Pantoja C, Echeverría-Ferrada F, Mellado-Arias M, Troncoso-Ramírez L. Percepción de influencias en la alimentación de personas mayores del sector Lan-C, comuna de Hualpén, Chile. Horiz Méd [Internet]. 2017 [citado 20 ene 2020]; 17(1): 45-50. Disponible en: http://www.scielo. org.pe/scielo.php?script=sci_arttext\&pid=S1727558X2017000100008\&lng=es.

9. Restrepo MSL, Morales GRM, Ramírez GMC, López LMV, Varela LLE. Los hábitos alimentarios en el adulto mayor y su relación con los procesos protectores y deteriorantes en salud. Rev Chil Nutr [Internet]. 2006 [citado 9 ene 2020]; 33(3): 500-10. Disponible en: http://dx.doi.org/10.4067/ S0717-75182006000500006

10. Aparicio García-Molina VA, Carbonell-Baeza A, Delgado-Fernández M. Beneficios de la actividad física en personas mayores. Rev int med cienc act fis deporte [Internet]. 2010 [citado 9 ene 2020]; 10(40): 556-576. Disponible en: https://www. redalyc.org/articulo.oa?id=54222122004

11. Encuesta Nacional de Salud y Nutrición. Informe final de resultados [Internet]. 2016 [citado 26 ene 2020]. Disponible en: https://www.gob.mx/cms/ uploads/attachment/file/209093/ENSANUT.pdf

12. Secretaría de Gobernación. Decreto por el que se reforman, adicionan y derogan diversas disposiciones del reglamento de la Ley General de Salud en Materia de Investigación para la Salud. Reforma [Internet]. 2014 [citado 2 abr 2020] Disponible en http://www.dof.gob.mx/nota detalle.php? codigo $=5339162 \&$ fecha $=02 / 04 / 2014$

13. Organización Mundial de la Salud. Aumentar el consumo de frutas y verduras para reducir el riesgo de enfermedades no transmisibles [Internet]. 2019 [citado 6 ene 2020]. Disponible en: https://www. who.int/elena/titles/fruit_vegetables_ncds/es/

14. Organización Mundial de la Salud. La importancia del consumo de frutas y verduras para tu salud [Internet]. 2013 [citado 13 nov 2019]. Disponible en: https:// alianzasalud.org.mx/2013/06/la-importancia-delconsumo-de-frutas-y-verduras-para-tu-salud/

15. Ramírez-Silva I, Rivera JA, Ponce X, Hernández-
Ávila M. Fruit and vegetable intake in the Mexican population: results from the Mexican National Health and Nutrition Survey 2006. Salud Públ Mex [Internet]. 2009 [citado 20 ene 2020]; 51(4): S574-S585. Disponible en: http://www.scielo.org. $\mathrm{mx} /$ scielo.php?script=sci_arttext $\&$ pid $=$ S003636342009001000012\&lng=es

16. VI Congreso Internacional de promoción al consumo de fruta y verdura, México/noticias VI congreso internacional / ' 5 al día' en Latinoamérica [Internet]. 2019 [citado 9 ene 2020]. Disponible en: https://www.5aldia.org/contenido-s.php?ro= $1166 \& \mathrm{sm}=1147 \& \mathrm{ag}=4767 \& \mathrm{co}=2503 \& \mathrm{pg}=1$

17. OMS. Dieta, nutrición y prevención de enfermedades crónicas. Informe de una consulta mixta FAO/OMS de expertos [Internet]. 2003 [citado 9 ene 2020]. Ginebra: OMS; Serie de Informes Técnicos 916. 152 p. Disponible en: https://www. who.int/nutrition/publications/obesity/WHO_ TRS_916_spa.pdf

18. Ramírez LM. Evaluación de la intervención nutricional sobre el riesgo nutricional y el apetito de un grupo de adultos mayores. Universidad del Valle de ATEMAJAC [Internet]. 2013 [citado 4 dic 2019]; 405-422. Disponible en: http://biblioteca. univa.mx/Anuario/2013/2013_17_evolucion_de_ la.pdf

19. Gaona-Pineda EB, Martínez-Tapia B, ArangoAngarita A, Valenzuela-Bravo D, Gómez-Acosta LM, Shamah-Levy T, et al. Consumo de grupos de alimentos y factores sociodemográficos en población mexicana. Salud Públ Mex [Internet]. 2018 [citado 9 ene 2020]; 60(3): 272-282. Disponible en: https://www.saludpublica.mx/index.php/ spm/article/view/8803

20. Barrón V, Rodríguez A, Chavarría P. Hábitos alimentarios, estado nutricional y estilos de vida en adultos mayores activos de la ciudad de Chillán, Chile. Rev chil Nutr [Internet]. 2017 [citado 9 ene 2020]; 44(1): 57-62. Disponible en: http://dx.doi. org/10.4067/S0717-75182017000100008

21. Rosner B, Gore R. Measurement Error Correction in Nutritional Epidemiology based on Individual Foods, with Application to the Relation of Diet to Breast Cancer. Am J Epidemiol [Internet]. 2001 [citado 20 ene 2020]; 154(9): 827-835. Disponible en: https://doi.org/10.1093/aje/154.9.827

22. Liu Q, Liao B, Tian Y, Chen Y, Luo D, Lin Y, Li $\mathrm{H}$, Wang KJ. Total, fluid consumption and risk of bladder cancer: a meta-analysis with updated data. Oncotarget [Internet]. 2017 [citado 9 ene 2020]; 8(33): 55467-55477. Disponible en: https://www. oncotarget.com/article/18100/text/

23. Negoianu D, Goldfarb S. Just Add Water. J Am Soc Nephrol [Internet]. 2008 [citado 9 ene 2020]; 
19: 1041-1048. Disponible en: http://dx.doi. org/10.1681/ASN.2008030274

24. Muñoz-Pérez MA, Zapater-Torras F. Impacto del cuidado de los nietos en la salud percibida y el apoyo social de las abuelas. Aten Primaria [Internet]. 2006 [citado 9 ene 2020]; 37(7): 37480. Disponible en: https://core.ac.uk/download/ pdf/82670968.pdf

25. Instituto Nacional de la Mujer. Las desigualdades de género vistas a través del estudio del uso del tiempo. Resultados de la Encuesta Nacional sobre uso del Tiempo, 2009, México. Situación de las personas adultas mayores en México [Internet]. 2010 [citado 27 nov 2020]. Disponible en: http://cedoc.inmujeres.gob.mx/documentos_ download/101243_1.pdf

26. Salgado AC. Revisión de estudios empíricos sobre el impacto de la religión, religiosidad y espiritualidad como factores protectores. Propósitos y Representaciones [Internet]. 2014 [citado 9 ene 2020]; 2(1): 121-159. Disponible en: http://dx.doi. org/10.20511/pyr2014.v2n1.55 